\title{
ON INFINITE PROLONGATIONS OF DIFFERENTIAL SYSTEMS ${ }^{1}$
}

\author{
H. H. JOHNSON
}

From any exterior differential system with independent variables, it will be shown how to construct, on an infinite dimensional space, an equivalent completely integrable differential system. Local solutions are discussed in the real or complex analytic case.

The calculus of exterior differential systems will be used freely $[1 ; 2]$. Prolongations are clearly described in [3]. All functions and forms are assumed infinitely differentiable unless otherwise restricted.

1. Infinite prolongations. Let $E^{n+m}$ be $n+m$-dimensional real/complex Euclidean space. Let $\Sigma$ be any real/complex infinitely differentiable closed exterior differential system with dependent variables $z^{1}, \cdots, z^{m}$ and independent variables $x^{1}, \cdots, x^{n}$, defined on an open subset $D$ of $E^{n+m}$.

The first prolongation, $\Sigma^{\prime}$, of $\Sigma$, can be accomplished as follows. Everywhere in $\Sigma$, replace $d z^{\lambda}$ by $\sum_{j=1}^{n} z_{j}^{\lambda} d x^{i}, \lambda=1, \cdots, m$, where $z_{j}^{\lambda}$ are $n m$ new variables. Using the exterior calculus, the coefficients of the independent terms in the resulting forms, together with the 0 forms of $\Sigma$, and the 1 -forms $d z^{\lambda}-\sum_{j=1}^{n} z_{j}^{\lambda} d x^{j}$, together generate $\Sigma^{\prime}$. ( $\lambda$ will always run from 1 to $m$, Latin letters from 1 to $n$; and the summation convention will be used.)

Thus, $\Sigma^{\prime}$ consists of

(1) a collection of 0 -forms or functions, $\left\{\Phi_{1}^{\alpha}\left(x^{i}, z^{\lambda}, z_{i}^{\lambda}\right) ; \alpha=1, \cdots, \alpha_{1}\right\}$ and their exterior derivatives, $\left\{d \Phi_{1}^{\alpha} ; \alpha=1, \cdots, \alpha_{1}\right\}$,

(2) the 1-forms $d z^{\lambda}-z_{j}^{\lambda} d x^{i}$ and their derivatives, $d z_{j \wedge}^{\lambda} d x^{j}$.

The second prolongation, $\Sigma^{\prime \prime}$, is obtained from $\Sigma^{\prime}$ by replacing $d z_{j}^{\lambda}$ with $z_{j k}^{\lambda} d x^{k}$, where the $z_{j k}^{\lambda}=z_{k j}^{\lambda}$ are $m n(n-1) / 2$ new variables. $\Sigma^{\prime \prime}$ consists of

(1) the 0 -forms of $\Sigma^{\prime},\left\{\Phi_{1}^{\alpha} ; \alpha=1, \cdots, \alpha_{1}\right\}$, and new functions $\left\{\Phi_{2}^{\alpha} ; \alpha=1, \cdots, \alpha_{2}\right\}$, together with the derivatives

$$
\left\{d \Phi_{2}^{\alpha} ; \alpha=1, \cdots, \alpha_{2}\right\},
$$

(2) $d z^{\lambda}-z_{j}^{\lambda} d x^{i}, d z_{j}^{\lambda}-z_{j k}^{\lambda} d x^{k}, d z_{j k \wedge}^{\lambda} d x^{k}$. It is important that $d \Phi_{1}^{\alpha}$ $\equiv 0$ modulo $\Sigma^{\prime \prime}, \alpha=1, \cdots, \alpha_{1}$, and $d\left(d z^{\lambda}-z_{j}^{\lambda} d x^{i}\right) \equiv 0$ modulo $\Sigma^{\prime \prime}$. The former is a consequence of the prolongation process, the latter of the symmetry $z_{j k}^{\lambda}=z_{k j}^{\lambda}$.

Received by the editors August 2, 1960.

${ }^{1}$ Research sponsored by OOR Contract DA-36-ORD-2164. 
$\Sigma^{(r)}$, the $r$ th prolongation, will be a closed system in $x^{i}, z^{\lambda}, \cdots$, $z_{i_{1}}^{\lambda} \cdots i_{q}$, where $q=1,2, \cdots, r$, and each $z_{i_{1}}^{\lambda} \cdots i_{q}$ is symmetric in the lower indices. $\Sigma^{(r)}$ consists of

(1) 0 -forms $\left\{\Phi_{1}^{\alpha} ; \alpha=1, \cdots, \alpha_{1}\right\},\left\{\Phi_{2}^{\alpha} ; \alpha=1, \cdots, \alpha_{2}\right\}, \cdots$, $\left\{\Phi_{r}^{\alpha} ; \alpha=1, \cdots, \alpha_{r}\right\}$, and 1-forms $\left\{d \Phi_{r}^{\alpha} ; \alpha=1, \cdots, \alpha_{r}\right\}$,

(2) $d z^{\lambda}-z_{j}^{\lambda} d x^{j}, \quad d z_{i_{1}}^{\lambda}-z_{i_{1} j}^{\lambda} d x^{j}, \cdots, d z_{i_{1}}^{\lambda} \cdots i_{r-1}-z_{i_{1}}^{\lambda} \cdots i_{r-1} j d x^{j}$, $d z_{i_{1}}^{\lambda} \cdots i_{r-1} j \wedge d x^{j}$.

Continued indefinitely, the result is an infinite sequence of 0 - and 1 -forms which may be written, grouping the 0 -forms together, as a system $\Sigma^{*}$ of

(1) 0 -forms $\psi^{\beta}\left(x^{i}, z^{\lambda}, \cdots, z_{i_{1}}^{\lambda} \cdots i_{q}, \cdots\right), \beta=1,2, \cdots$, each depending on an only finite number of variables,

(2) $d z^{\lambda}-z_{j}^{\lambda} d x^{j}, \cdots, d z_{j_{1}}^{\lambda} \cdots j_{q}-z_{j_{1}}^{\lambda} \cdots j_{q} j d x^{j}, \cdots$. The $z_{j_{1}}^{\lambda} \cdots j_{q}$ are symmetric in lower indices and may assume any real/complex values, while the $\left(x^{i}, z^{\lambda}\right) \in D$. Each $\psi^{\beta}$ is infinitely differentiable. Most important, $\Sigma^{*}$ is completely integrable, i.e., for any 0 - or 1 -form $\omega \in \Sigma^{*}$, there is a finite subset, $\Sigma_{\omega}^{*}$ of $\Sigma^{*}$, such that

$$
d \omega \equiv 0 \text { modulo } \Sigma_{\omega}^{*} \text {. }
$$

A solution of $\Sigma^{*}$ consists of infinitely differentiable functions $z^{\lambda}\left(x^{i}\right), \cdots, z_{t_{1}}^{\lambda} \cdots i_{q}\left(x^{i}\right), \cdots$, defined on a neighborhood $N$ in $E^{n}$, so that $\left(x^{i}, z^{\lambda}\left(x^{i}\right)\right) \in D$ when $\left(x^{i}\right) \in N$, and which causes every form in $\Sigma^{*}$ to vanish identically when $z^{\lambda}, d z^{\lambda}, z_{i_{1}}^{\lambda} \cdots i_{q}$, and $d z_{i_{1}}^{\lambda} \cdots i_{q}$ are replaced by $z^{\lambda}\left(x^{i}\right), d z^{\lambda}\left(x^{i}\right)$, the differential, $z_{i_{1}}^{\lambda} \cdots i_{q}\left(x^{i}\right)$, and $d z_{i_{1}}^{\lambda} \cdots i_{q}\left(x^{i}\right)$, respectively. Vanishing of the forms in (2) in $\Sigma^{*}$ is equivalent to

$$
\frac{\partial^{\alpha} z^{\lambda}\left(x^{i}\right)}{\partial x^{j_{1}} \cdots \partial x^{i_{q}}}=z_{j_{1} \cdots j_{q}}^{\lambda}\left(x^{i}\right) \text {. }
$$

There is a one-to-one correspondence between the solutions of $\Sigma$ and $\Sigma^{(r)}$ [3], and it is easy to see that this property extends to $\Sigma^{*}$, the correspondence being given by (A).

2. Analytic case. Assume that the forms in $\Sigma$ are real/complex analytic, i.e., all functions appearing as coefficients have this property. Then all forms in $\Sigma^{*}$ are analytic. Let $\left(j_{1}, \cdots, j_{q}\right)$ be a sequence of $q$ integers, $1 \leqq j_{k} \leqq n$. If, among these, there are exactly $\rho_{1}$ ones, $\rho_{2}$ twos, $\cdots$, and $\rho_{n} n$ 's, let $\rho\left(j_{1}, \cdots, j_{q}\right)=\rho_{1} ! \rho_{2} ! \cdots \rho_{n}$ !.

A sequence $\left(u^{i}, v^{\lambda}, \cdots, v_{j_{1}}^{\lambda} \cdots j_{q}, \cdots\right)$ of real/complex numbers, where the $v_{j_{1}}^{\lambda} \ldots j_{q}$ are symmetric in the lower indices, is called a zero of $\Sigma^{*}$ if, for all $\beta, \psi^{\beta}\left(u^{i}, v^{\lambda}, \cdots, v_{j_{1}}^{\lambda} \cdots j_{q}, \cdots\right)=0$.

Theorem. Let $\left(u^{i}, v^{\lambda}, \cdots, v_{j_{1}}^{\lambda} \cdots j_{q}, \cdots\right)$ be a zero of $\Sigma^{*}$, where 


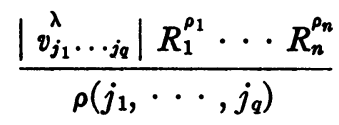

is a bounded sequence for some positive real numbers $R_{1}, \cdots, R_{n}$, and $\rho\left(j_{1}, \cdots, j_{q}\right)=\rho_{1} ! \cdots \rho_{q} !$. Then there exists a unique solution,

$$
z^{\lambda}\left(x^{i}\right), \cdots, z_{j_{1} \cdots j_{q}}^{\lambda}\left(x^{i}\right), \cdots
$$

of $\Sigma^{*}$ defined in a neighborhood of $\left(u^{i}\right)$ and satisfying the initial conditions

$$
z^{\lambda}\left(u^{i}\right)=v^{\lambda}, \cdots, z_{j_{1}}^{\lambda} \cdots j_{q}\left(u^{i}\right)=v_{j_{1} \cdots j_{q}}^{\lambda} .
$$

Proof. Let

$$
a_{\rho_{1} \cdots \rho_{n}}^{\lambda}=\frac{1}{\rho_{1} ! \cdots \rho_{n} !} v_{i_{1} \cdots i_{\rho_{1}}+\cdots \rho_{n}}^{\lambda},
$$

where $\left(i_{1}, \cdots, i_{\rho_{1}+\cdots+\rho_{n}}\right)$ consists of $\rho_{1}$ ones, $\rho_{2}$ twos, $\cdots$, and $\rho_{n} n$ 's, if not all these are zero, and $a_{0 \cdots 0}^{\lambda}=v^{\lambda}$. Let

$$
z^{\lambda}\left(x^{j}\right)=\sum_{\rho_{1}=0}^{\infty} \cdots \sum_{\rho_{n}=0}^{\infty} a_{\rho_{1}}^{\lambda} \ldots \rho_{n}\left(x^{1}-u^{1}\right)^{\rho_{1}} \cdots\left(x^{n}-u^{n}\right)^{\rho_{n}}
$$

$$
\hat{z}_{j_{1} \cdots j_{q}}\left(x^{i}\right)=\frac{\partial^{q} z^{\lambda}\left(x^{i}\right)}{\partial x^{j_{1}} \cdots \partial x^{j_{q}}} .
$$

These functions converge for $\left|x^{i}-u^{j}\right|<R_{j}$, satisfy the initial conditions, and cause the 1-forms $d z^{\lambda}-z_{j}^{\lambda} d x^{j}, \cdots, d z_{j_{1} \cdots j_{q}}^{\lambda}-z_{j_{1} \ldots j_{q}}^{\lambda} d x^{j}$, ... to vanish.

Let $\psi^{\beta}\left(x^{i}\right)$ denote $\psi^{\beta}$ after $z^{\lambda}, \cdots, z_{j_{1} \cdots g_{q}}^{\lambda}, \cdots$ are replaced by the corresponding functions (B). By hypothesis, $\psi^{\beta}\left(u^{i}\right)=0$. Since $\Sigma^{*}$ is completely integrable,

$$
d \dot{\psi}^{\beta} \equiv 0 \text { modulo } \Sigma^{*}
$$

hence,

$$
\frac{\partial \psi^{\beta}}{\partial x^{j}}+\frac{\partial \psi^{\beta}}{\partial z^{\lambda}} z_{j}^{\lambda}+\cdots+\frac{\partial \psi^{\beta}}{\partial z_{j_{1}}^{\lambda} \cdots j_{q}} z_{j_{1} \cdots j_{q} j}^{\lambda}+\cdots \equiv 0 \text { modulo }\left\{\psi^{\beta}\right\},
$$

and the sums on both sides are finite. Hence, there are analytic functions $A_{j \gamma}^{\beta}\left(x^{i}\right)$ such that

$$
\frac{\partial \Psi^{\beta}}{\partial x^{i}}=A_{j \gamma}^{\beta} \Psi^{\gamma}
$$


Therefore, $\left(\partial \Psi^{\beta} / \partial x^{j}\right)\left(u^{i}\right)=0$, all $\beta$. In the same way

$$
\frac{\partial^{2} \Psi^{\beta}}{\partial x^{j} \partial x^{k}}=A_{j \gamma}^{\beta} \frac{\partial \Psi^{\gamma}}{\partial x^{k}}+\frac{A_{j \gamma}^{\beta}}{\partial x^{k}} \Psi^{\gamma},
$$

implies $\left(\partial^{2} \psi^{\beta} / \partial x^{j} \partial x^{k}\right)\left(u^{i}\right)=0$, etc. Thus, the Taylor's expansion of $\psi^{\beta}$ at $\left(u^{i}\right)$ is zero. The initial conditions thus determine uniquely the $z^{\lambda}\left(x^{i}\right)$, hence the solution. Q.E.D.

\section{BIBLIOGRAPHY}

1. E. Cartan, Les systèmes différentiels extérieurs et leurs applications géométriques, Paris, Hermann, 1945.

2. E. Kähler, Einführung in die Theorie der Systeme von Differentialgleichungen, Hamburger Math. Einzelschrifte Heft 16, Leipzig, B. G. Teubner, 1934.

3. M. Kuranishi, On E. Cartan's prolongation theorem of exterior differential systems, Amer. J. Math. vol. 79 (1957) pp. 1-47.

Princeton University 